\title{
Assessment of Sero-prevalence of HIV and Associated Factors Among Debre Berhan University Students, Ethiopia
}

\author{
Yohannes Girma Zewdie ${ }^{1}$, Alemnesh Tesema Tilahun², Biniam Assefa Alamirew ${ }^{1}$ \\ ${ }^{1}$ Surgery Department, College of Medicine, Debre Berhan University, Debre Berhan, Ethiopia \\ ${ }^{2}$ Department of Medical Laboratory Science, College of Medicine, Debre Berhan University, Debre Berhan, Ethiopia
}

Email address:

jhngirma@gmail.com (Y. G. Zewdie), aleminat@gmail.com (A. T. Tilahun), binishashasha@gmail.com (B. A. Alamirew)

\section{To cite this article:}

Yohannes Girma Zewdie, Alemnesh Tesema Tilahun, Biniam Assefa Alamirew. Assessment of Sero-prevalence of HIV and Associated Factors Among Debre Berhan University Students, Ethiopia. Clinical Medicine Research. Vol. 8, No. 2, 2019, pp. 47-52.

doi: $10.11648 /$ j.cmr.20190802.13

Received: August 10, 2018; Accepted: November 22, 2018; Published: July 11, 2019

\begin{abstract}
Introduction: Young adults including university students are at high risk of acquiring HIV due to their risky sexual practices and the recent emergence of rising HIV epidemic in Ethiopia. The aim of this study was to assess Sero-prevalence of HIV and associated factors among students of Debre Berhan University. Method: Institution based Cross sectional study design was employed. Sample size was determined using Epi Info version 3.4 which is 580. A combination of stratified and simple random sampling technique was carried out to allocate the sample. Data was collected by self-administered questionnaire and blood sample for HIV screening was taken. Data entry and analysis was done using SPSS version 16. 95\% C.I was used to determine the associated factors of risky sexual behavior and P-value less than 0.05 was considered to be significant. Result: The HIV Sero-prevalence among the students was 0.36\%. Among 557 participants, $140(25.13 \%)$ reported having at least one risky sexual behavior in their lifetime. Participants, 140 (25.13\%) reported having at least one risky sexual behavior in their lifetime. Cigarette smoking and pornography exposure shows significant association with risky sexual behavior. Therefore, this study suggested that HIV Sero-prevalence and risky sexual behavior among the students is relatively high and needs urgent interventions.
\end{abstract}

Keywords: Higher Institution, Knowledge, Peer, Risky Behavior, Substance Use, HIV

\section{Introduction}

Human Immunodeficiency Virus (HIV) infection has been one of the top health related challenges for the past three decades. This is especially imperative for the African nations which bear $67 \%$ of the world's HIV/AIDS burden (World Health Organization WHO, 2010). Risky sexual behaviors contribute to the high HIV prevalence in Africa. Certain aspects of social life place members of higher education institutions are at risk of contracting with HIV. Enhanced personal freedom coupled with the attractions and pressures of life in higher education institutions is a recipe for sexual activity and experimentation. Moreover, College students are at a high risk because they tend to be sexually adventurous, often with multiple partners and do not consistently use condoms. Besides, commercial sex may not be uncommon as poor students seek to earn money to pay for personal upkeep [1].

Research indicated that in the world $40-50 \%$ university students had multiple sexual partners and $30-60 \%$ of them do not use condom during last sex. Eight percent had sex with commercial sex worker or sex for money/gift [2]. In Ethiopia among sexually active university students $37.5 \%$ of them had multiple sexual partners and $29 \%$ do not use condom during their last sexual contact. In addition, fifteen percent had sex because of exchange of money/gift [3].

A number of studies have showed that AIDS (acquired Immuno deficiency syndrome) has progressively been on the increase and constitutes a big problem among college and university students, although the extent of the problem is relatively unknown [1, 4-5]. Though studies have been done on general populations [EDHS 2011], studies on specific populations such as institutions of higher learning are scanty, in particular, Sero-prevalence data on this target group is lacking in Ethiopia.

By recognizing the above facts, the researchers want to 
assess HIV sero-prevalence and sexual behaviors among these youth generation. HIV testing is beneficial to better plan all aspect of their future. Understanding these risk factors and prevalence of HIV can help health care practitioners and health educators to develop messages and interventions to reduce young peoples' risk of infection with HIV and other STDs (sexually transmitted diseases). Therefore, the main objective of this study was to assess HIV Sero-prevalence and associated factors among regular undergraduate DBU students in the period of March 2017 to April 2017 G.C.

\section{Methods}

\subsection{Study Design and Period}

In this research, Institution based cross-sectional quantitative survey was undertaken. Simple random sampling which is a probability sampling method was applied to select respondents of the research. The sample size of the research was 580 undergraduate regular students. The study period was from March 1/2017- April 30/2017G.C.

\subsection{Study Area and Population}

\subsubsection{Study Area}

DBU which is found in Debre Berhan city, $130 \mathrm{~km}$ from the capital Addis Ababa, is a public educational institution established in 1999 E.C and currently, the enrolment has significantly increased to more than 24,000 students in the regular, extension, summer and distance programs in 42 undergraduate, 23 postgraduate and one $\mathrm{PhD}$ programs under 10 colleges and 2 institutes.

\subsubsection{Source Population}

All regular students of DBU.

\subsubsection{Study Population}

All selected faculties' regular students of DBU during the study period.

\subsection{Inclusion Criteria and Exclusion Criteria}

\subsubsection{Inclusion Criteria}

All selected faculties' regular students of DBU during the study period.

\subsubsection{Exclusion Criteria}

A student who is unable to communicate because of ear disability.

All summer, extension and distance students.

\subsection{Sample Size Determination and Sampling Procedure}

\subsubsection{Sample Size Determination}

Sample size was determined by using the assumption of single population proportion formula by using Epi Info version 3.4 sample size calculation methods. The final sample size calculated was 580 .

\subsubsection{Sampling Procedure}

A combination of stratified and simple random sampling technique was carried out to allocate the sample proportionally to the size of students in each faculty. The faculty then further stratified by year of study (year 1-5). Finally simple random sampling was employed to select study subjects from the sample frame.

\subsection{Data Collection Procedures and Tool}

Data collection was done by two senior laboratory technicians and three trained voluntary counseling and testing (VCT) nurses. Pretest was done on $5 \%$ of total sample size of the study subjects in DBU Student Clinic to ensure the reliability and internal consistency of the questionnaire and the quality of rapid viral detection kits. The kit has known positive and known negative controls and the time each test took and the color change occurred was assessed. Intermediate results were further tested using next confirmatory steps with the respective reagents. The questionnaire was adapted from other similar studies and Behavioral surveillance survey (BSS) questionnaires, which included demographic characteristics, sexual behaviors, risk assessment, and knowledge on HIV/AIDS and institutional factors. A number ranging from Y001 to Y580 was written immediately after each VCT both on the questionnaire and kit. At the end of each self-administered questionnaire, a drop of blood was collected by the technicians using sterile technique. Upon completion of collection, each blood specimen was detected for viral reactivity. A Modified Algorithm technique for HIV rapid testing was carried out following its standard procedures. Results were recorded reactive or non-reactive and post-test counseling were given after the test result.

\subsection{Variables}

\subsubsection{Outcome Variables}

(1) Risky sexual behavior

(2) Sero prevalence of HIV

\subsubsection{Independent Variables}

Socio demographic characteristics, knowledge, HIV/AIDS Sero-status, substance use, sexual history.

\subsection{Operational Definitions}

(1) Comprehensive knowledge of HIV/AIDS transmission: means knowing HIV transmission method and rejecting twomajor misconceptions, HIV transmission by mosquito biteand from eating raw meat prepared by a person infected withHIV.

(2) Comprehensive knowledge of HIV prevention method: means knowing both abstaining from sexual intercourse, condom use, and limiting sex partner to uninfected partnersprevents HIV.

(3) HIV Sero-status: Is either being Sero Positive or Negative for HIV testing.

(4) Substances: Any non-medical drugs used by study 
subjects such as alcohol, khat, tobacco, Cannabis, heroin, cocaine, and marijuana to alter their mood or behavior.

(5) Risky Sexual behavior: In this study it is defined as one of the following: not using condom (inconsistent use of condoms), having multiple sexual partner, starting sex before age 15 years and sex with commercial sex workers.

\subsection{Data Processing and Analysis}

Data entry, clearance was employed and analyzed by using SPSS, version 16, statistical software. Statistical tests were carried out for risky sexual behavior and its associated variables. For each independent variable, frequencies, odds ratio at $95 \%$ C.I and P-value at alpha 0.05 was calculated. Multivariate analysis was computed to control the effect of confounders. Interpretation and inferences were made and results were presented in tables.

\subsection{Data Quality Control and Management}

In order to assure the data quality the study used standard tool, training, close supervision, use of expertise as data collector, pre-tested in the respective sample collection and detection.

\subsection{Ethical Clearance}

Ethical clearance was obtained from the DBU research and publication office. A verbal consent was obtained from the study participants.

\section{Results}

\subsection{Socio-demographic Characteristics}

Among the study sample, a total of 557 study subjects were participated in the study with $96 \%$ of response rate. With respect to their residence, 310 (55.7\%) dwell in urban. By ethnicity, Amhara and Oromo constituted $451(81 \%)$ and $38(6.8 \%)$ respectively followed by $30(5.4 \%)$ Tigrai. As of religion, 503(90.3\%) and 27(4.8\%) were Orthodox Christians and Muslims respectively, followed by Protestants 20(3.6\%). About gender, 404 (72.5\%) males and $153(27.5 \%)$ were females. Among the participants, $458(82.2 \%)$ were in the age group $20-24$ years old. The mean ages of the participant were 21.78 year (S.D \pm 2.690 ) with a range between 18-43 years. Majority $516(92.6 \%)$ of the study participant were unmarried. In perspective of family income, 364(65.4\%) were reported to have a medium, 146(26.2\%) low and $11(2 \%)$ high income respectively. Most of the students' 491(88.2\%) were from non-health related fields.

\subsection{HIV Sero-prevalence Among Debre Berhan University Students}

Among voluntarily tested 557 students $0.36 \%$ (2) was identified to be HIV Sero positive. Assuming that HIV in Ethiopia is commonly transmitted by heterosexual contact; the prevalence of HIV among sexually active students was $1 \%(2 / 200)$. All prevalence variation among different variables is not statistically significant.

\subsection{Risks for STIS/HIV Infection}

Of the total study participants, 201 (36.1\%) of the students were sexually active (male; $78.1 \% \mathrm{Vs}$ females; $21.9 \%$ ). The mean age at first sexual intercourse was $18.1( \pm 2.2)$ years. Of the total sexually active students $(66.2 \%)$ had initiated sex before joining university. Of the sexually active students, $74.2 \%$ have risks for STIs/HIV infection. Majority (62.5\%) of sexually active students gave falling in love as a reason to initiate sex, $23 \%$ due to peer pressure and $5.5 \%$ to get money or other benefits from their sexual partners. The prevalence of condom use among sexually active individuals was low $(45.8 \%)$. The main reason for not using condom being never thought about it (42.48\%), hurry for sex $(27.4 \%)$ and no opportunity to use condom $(18.6 \%)$. Knowledge of the majority of students $(75.3 \%)$ regarding HIV transmission, prevention was good. The magnitude of multiple sexual partner among sexually active students was $44.8 \%$.

\subsection{Substance Use Pattern and Pornography Exposure}

The most widely used substance was alcohol (12.7\%), illicit drug use (11.1\%) and cigarette smoking (9.2\%). For most of the students they started alcohol drinking before joining university (59.2\%), but illicit drug use and cigarette smoking were started after joining university $(70.5 \%$ and $66.7 \%$ respectively). The most commonly used illicit drug was Khat (59\%) followed by Hashish (18\%). The prevalence of pornography exposure was $24.4 \%$. Major means of pornography exposure was through the internet (46.3\%), video cassette $(27.9 \%)$ and pictures $(25.7 \%)$. The reason to initiate pornography was peer pressure $(43.4 \%)$, easy internet access (42.6).

\subsection{Factors Associated with Risky Sexual Behaviors}

Multivariate analysis showed that, cigarette smoking and pornography exposure had significant association with risky sexual behavior. Students who smoke cigarette were 5.66 times more likely to engage into risky sexual behavior than students who don't s moke (OR $[95 \% \mathrm{CI}]=5.662[1.115$ 28.76]) and those students who had pornography exposure were 3.13 times more likely to engage into risky sexual behaviors than those who don't had pornography exposure $(\mathrm{OR}[95 \% \mathrm{CI}]=3.132[1.287-7.621])($ Table 1$)$.

Table 1. Multivariate analysis risky sexual behavior by factors affecting risky sexual behavior of Debre Berhan university students, March, 2017.

\begin{tabular}{|c|c|c|c|c|c|c|}
\hline Variables & Category & risky & Non risky & COR $(95 \%$ CI) & P value & AOR (95\% CI) \\
\hline age category & $\begin{array}{l}15-19 \\
20-24 \\
>25(\mathrm{R})\end{array}$ & $\begin{array}{l}5(2.58) \\
113(58.23) \\
26(13.4)\end{array}$ & $\begin{array}{l}1(0.52) \\
36(18.56) \\
13(6.7)\end{array}$ & $\begin{array}{l}2.5(0.264-23.669) \\
1.569(0.731-3.371) \\
1\end{array}$ & $\begin{array}{l}0.424 \\
0.248\end{array}$ & $\begin{array}{l}3.319(0.305-36.164) \\
1.157(0.467-2.855) \\
1\end{array}$ \\
\hline
\end{tabular}




\begin{tabular}{|c|c|c|c|c|c|c|}
\hline Variables & Category & risky & Non risky & COR $(95 \%$ CI $)$ & P value & AOR (95\% CI) \\
\hline \multirow{5}{*}{ Year of study } & $1^{\text {st }}$ & $21(10.82)$ & $8(4.12)$ & $0.563(0.169-1.868)$ & 0.347 & $0.348(0.085-1.423)$ \\
\hline & $2^{\text {nd }}$ & $34(17.53)$ & $19(9.79)$ & $0.383(0.135-1.091)$ & 0.072 & $0.289(0.088-0.957)$ \\
\hline & $3^{\text {rd }}$ & $51(26.29)$ & $13(6.7)$ & $0.841(0.288-2.455)$ & 0.751 & $0.51(0.146-1.782)$ \\
\hline & $4^{\text {th }}$ & $10(5.15)$ & $4(2.06)$ & $0.536(0.125-2.299)$ & 0.401 & $0.385(0.071-2.094)$ \\
\hline & $5^{\text {th }}(\mathrm{R})$ & $28(14.43)$ & $6(3.09)$ & 1 & & 1 \\
\hline \multirow{2}{*}{$\begin{array}{l}\text { Belief of Curative } \\
\text { medicine for HIV }\end{array}$} & Yes & $92(47.42)$ & $38(19.59)$ & $0.559(0.269-1.162)$ & 0.119 & $0.9(0.367-2.207)$ \\
\hline & No (R) & $52(26.8)$ & $12(6.18)$ & 1 & & 1 \\
\hline \multirow{2}{*}{ Ever acquired STI } & Yes & $24(12.37)$ & $3(1.55)$ & $3.133(0.901-10.901)$ & 0.073 & $2.097(0.443-9.928)$ \\
\hline & No (R) & $120(61.86)$ & $47(24.22)$ & 1 & & 1 \\
\hline \multirow{2}{*}{ Regular alcohol use } & Yes & $43(22.16)$ & $9(4.64)$ & $1.939(0.867-4.338)$ & 0.107 & $0.636(0.196-2.061)$ \\
\hline & No (R) & $101(52.06)$ & $41(21.13)$ & 1 & & 1 \\
\hline \multirow{2}{*}{ Regular cigarette smoke } & Yes & $37(19.07)$ & $3(1.55)$ & $5.417(1.591-18.452)$ & 0.007 & $5.662(1.115-28.76)$ \\
\hline & No (R) & $107(55.15)$ & $47(24.22)$ & 1 & & 1 \\
\hline \multirow{2}{*}{$\begin{array}{l}\text { Regular use of illicit } \\
\text { drugs }\end{array}$} & Yes & $40(20.62)$ & $7(3.61)$ & $2.363(0.982-5.685)$ & 0.055 & $0.51(0.12-2.165)$ \\
\hline & No (R) & $104(53.61)$ & $43(22.16)$ & 1 & & 1 \\
\hline \multirow{2}{*}{ Watch pornography } & Yes & $74(38.14)$ & $14(7.22)$ & $2.718(1.352-5.466)$ & 0.005 & $3.132(1.287-7.621)$ \\
\hline & No $(R)$ & $70(36.08)$ & $36(18.56)$ & 1 & & 1 \\
\hline
\end{tabular}

\section{Discussion}

\section{Risky sexual behaviors}

The mean age of sexual initiation (18.1 years) were comparable with other studies done among youths in Haramaya University (HU), MadaWalabu University (MWU), Dire Dawa town and Addis Abeba University (AAU) students [6-9]. In this study, 36.1\% of the students had sexual experience $(78.5 \%$ for males and $21.5 \%$ for females). This result is similar to the study among AAU and Arbaminch University (AMU) students [9-10].

The prevalence of life time multiple sexual partners was $44.8 \%$. The proportion of life time multiple sexual partners are by far greater than the findings in HU (35.4\%), MWU (30.4\%) and Bahir Dar University (BDU) (27.8\%). The figurative -difference could be due to the difference in sample size and comprehensive university based behavioral change interventions in the above mentioned universities. But a study in Wolaita Sodo University (WSU) has reported life time multiple sexual partner rate of 70.6\%. [7-8, 11-12].

In our study, $45.8 \%$ of the students practiced unprotected sexual intercourse in the last 12 months. This rate of unprotected Sexual practice is similar with the finding in MWU (44.0\%) but higher than BDU (34.4\%). However, the rate of unprotected sex with casual partners in Jimma University looks somehow greater $(56.3 \%)$ [8, 11, 13]. This difference might be attributed to socio-demographic and sample size differences between the participants and studies respectively. Several reasons for not using condoms were raised, ranging from being never thought about it $(42.5 \%)$, a hurry to have sex $(27.4 \%)$ and not having condoms (18.6\%). The reasons given concur with the findings of Jijiga [14].

In this study, $4.7 \%$ of sexually active male students reported ever had history of sexual contact with commercial sex workers (CSW). Slightly higher result (7.8\%) of students in BDU(10), reported sexual contact with commercial sex workers while a study in HU and MWU reported that $39.9 \%$ and $24 \%$ of sexually active male student had sexual contact with commercial sex workers respectively [7-8]. The low level of sex with commercial sex worker in this study might be attributed to the lesser number of night clubs and drug house available in the area.

About $25.13 \%$ of the students were engaged in at least one of the risky sexual behaviors which accounts for $78.5 \%$ for males and $21.5 \%$ for females. This study is lower when compared with the study conducted in HU students, where $65.8 \%$ of them had at least one of the risky sexual behaviors [7]. The difference between the above studies may be due to difference in usage of substances that initiate risky sexual behaviors like khat chewing and alcohol abuse between the two settings.

Multivariable analysis showed that cigarette smoking and pornography exposure has a significant association with a risky sexual behavior. These findings were comparable with study conducted in HU, Dire Dawa town, Jimma University and AAU $[7,9,13,15]$. This might be due to the nature of substances in altering rational decision making ability, decreasing inhibitions, and increase risk taking behavior.

The HIV prevalence in this study among sexually active students was $1 \%$. This HIV prevalence in DBU students is comparable to national prevalence (EDHS, 2014). This indicates sexually active University students in Ethiopia are at risk for HIV. The study in different part of the world ranges from among $0.2 \%$ in USA and $-7.7 \%$ South Africa [16]. The HIV prevalence in our University is lower than other African Universities students. The variation might be because of national difference in HIV prevalence. As compared to many sub-Saharan Africa (SSA) countries, HIV prevalence in Ethiopia is very low [EDHS, 2014]. The other possible reason for low prevalence of HIV among our students might be majority of the students were practicing sexual contact with fellow students. Even though the prevalence is lower than other African University students, $1 \%$ prevalence of HIV has a great implication of Public health importance.

Most of the respondents are clear on the major ways of HIV transmission but they have a gap on knowledge of HIV transmission misconceptions. This finding is similar with the study done in Ambo University [17]. In this study knowledge on HIV has no association to risky sexual behavior, this goes with the research done in central Florida and Bhutan [18-19]. 
However this is contradictory to the finding in Ambo University and St Marry University [17, 20].

The overall prevalence of "ever used drug" for at least one "drug" is $33 \%$. This is lower than a similar study on HU (53.8) [7]. However, it is higher than other study conducted in Bahir Dar City private College [21]. The difference might be due to life or living situation of the study respondents. It is apparent from the lifetime prevalence data that few students had tried illicit drugs. This might be due to students didn't get these illicit drugs easily, and the possession and use of these drugs results in penalty under the law of the country.

\section{Conclusion}

Comparably, lower prevalence of HIV was noticed in the study $(0.36 \%)$. In this study about $25.13 \%$ of the students were engaged in at least one risky sexual behavior. The use of substances especially cigarette smoking and pornography exposure were significantly associated with risky sexual behavior. Knowledge about HIV has no association with risky sexual behavior and this needs other factors to be investigated. Knowledge of the respondents were generally unsatisfactory especially on the HIV transmission misconceptions.

\section{Recommendations}

DBU should strength student clubs, design and implement awareness rising and risk reduction activities and promote peer education. Students should be encouraged to practice premarital abstinence, improve accessibility of condom and point of delivery.

\section{Acknowledgements}

We would like to thank our almighty God who help us in all things. We would like to extend our thanks to the gender and cross cutting of issues directorate of Debreberhan University for financing the study. Our special thanks and sincere appreciation also goes to Debreberhan University administration and health center staffs as well as supervisors and study participants for contribution to the success of data collection. Finally, we would like to forward our gratitude to staffs of the Department of Public Health, student clinic staffs, my friends and my family for their support and contribution in the study.

\section{Conflicts of Interest}

All the authors do not have any possible conflicts of interest.

\section{References}

[1] Teka T (1993). College Students' Attitude and knowledge of AIDS. Ethiop. Med. J. 314: 233-237.
[2] Salameh P (2009) Knowledge and practice of university students in Lebanon regarding contraception, eastern Mediterranean health journal, Vol. 15, No. 2,

[3] Nigatu \& Seman (2011). Attitudes and practices on HIV preventions among students of higher education institutions in Ethiopia: The case of Addis Ababa University. International Research JournalsVol. 2 (2) pp. 828-840.

[4] Tefera B, Challi J, and Yoseph M (2004). KAP about HIV/AIDS, VCT Among students of Jimma University, Jimma Zone, SW, Ethiopia. Ethiop. J. Health Sci. Vol. 14, Specal issue.

[5] Getinet T (2009). Self-reported sexual experiences, sexual conduct and safer sex practices of AAU undergraduate male and female student's on the context of HIV/AIDS pandemics, A PhD dissertation the State University of New York at Buffalo, USA.

[6] Agency CS. Ethiopia demographic health survey. Addis Abeba; 2017.

[7] Derese A, Seme A, Misganaw C. Assessment of substance use and risky sexual behaviour among Haramaya University Students, Ethiopia. Sci J Public Heal. 2014; 2 (2): 102-10.

[8] Mengistu TS, Melku AT, Bedada ND, Eticha BT. Risks for STIs / HIV infection among Madawalabu University students, Southeast Ethiopia: a cross sectional study. Reproductive Health Journal. 2013; 10 (1): 1-7.

[9] Alemu H. Assessment of factors contributing to voluntary counselling and testing (VCT) utilization among youth in Dire Dawa Administrative Council By Acknowledgments. Addis Abeba; 2008. Health. 2011; 8 (2): 141-54.

[10] Soboka B, Kejela G. Assessment of Risky Sexual Behaviors among Arba Minch University Students, Arba Minch Behavior Assessment of Risky Sexual Behaviors among Arba Minch University Students,. J Child Adolesc. 2015; 3 (2).

[11] Substance Use, Sexual Orientation and Risky Sexual Behaviors for HIV infection Among Bahir Dar University Students. North-West Ethiopia [Masters thesis]. Jimma University; 2010.

[12] T G. Predictors of HIV Risk Preventing Behaviors among Wolaita Soddo University Students. Sodo, South Ethiopia [Masters thesis]. Jimma University; 2010.

[13] Tefera B, Challi J YM. Knowledge, attitude and practice about HIV/ AIDs, voluntary counseling and testing among students of Jimma University, Jimma zone, Southwest Ethiopia. Ethiop J Heal Sci. 2004; 14: 43-53.

[14] Mavhandu-mudzusi AH, Asgedom T. The prevalence of risky sexual behaviours amongst undergraduate students in Jigjiga University, Ethiopia. Heal SA Gesondheid. 2016; 21: 179-86.

[15] Regassa N, Kedir S. Attitudes and practices on HIV preventions among students of higher education institutions in Ethiopia: the case of Addis Ababa University. East Afr J Public Health. 2011; 8 (2): 141-54.

[16] A. Kharsany (2012) HIV prevalence among high school learners - opportunities for schools-based HIV testing programs and sexual reproductive health services BMC Public HealthVol-12; 1: 231. 
[17] Sahile Z, Mekuria M, Yared A. Comprehensive HIV / AIDS Knowledge and Sexual Behavior among University Students in Ambo, Central Ethiopia: Implication to Improve Intervention. J Sex Transm Dis. 2015; Volume 201: 1-6.

[18] Castora M. An Assessment of University Students ' Knowledge, Attitudes, and Behaviors toward Sex. Undergrad Res J. 2005; 1: 28-37.

[19] Norbu K, Mukhia S, Tshokey. Assessment of knowledge on sexually transmitted infections and sexual risk behaviour in two rural districts of Bhutan. BMC Public Health. 2013; 13: $2-7$.

[20] Shewangizaw Z, Girma M, Endazenaw G. Assessment of HIV Sero-Prevalence among St. Marry University Students, Ethiopia. Sci J Public Heal. 2015; 3 (4): 468-77.

[21] Alamrew Z, Bedimo M, Azage M. Risky Sexual Practices and Associated Factors for HIV / AIDS Infection among Private College Students in Bahir Dar City, Northwest Ethiopia. ISRN PublicHealth. 2013; Volume 201. 